\title{
A comparative study of CHN-MNC, GA and PSO for solving constraints satisfaction problems
}

\author{
Bouhouch adil ${ }^{1}$, Loqman Chakir ${ }^{2}$, Bennis Hamid ${ }^{3}$ and El Qadi Abderrahim ${ }^{4}$ \\ \{bouhouch.adil@gmail.com ${ }^{1}$ \} \\ Department of Computer Science, Faculty Sciences, Chouaib Doukkali University, El Jadida, \\ Morocco ${ }^{1}$ \\ Department of informatics, Faculty Sciences, Dhar Mehraz, Sidi Mohammed Ben Abdellah Fez, \\ Morocco $^{2}$ \\ Team TIM, High School of Technology - Moulay Ismail University, Meknes, Morocco ${ }^{3}$ \\ High School of Technology - Mohammed V University of Rabat Morocco ${ }^{4}$
}

\begin{abstract}
Our approach CHN-MNC, based Continuous Hopfield neural network and Min-Conflict heuristic), have proved that is more efficient than using $\mathrm{CHN}$ alone to solve Constraints Satisfaction Problem (CSP). In This paper we study the performance of CHN-MNC by comparing it robustness with two evolutionary algorithms. We choose a Genetic Algorithm and Swarm optimisation to performers this study. Some numerical experiments are done over a variety of problems to verify the efficiency and fast convergence of our approach. abstract needs to summarize the content of the paper.
\end{abstract}

Keywords: CSP, Metaheuristics, GA, PSO, Min-Conflict Heuristic.

\section{Introduction}

A large number of real world problems can be represented as constraint satisfaction problems (CSP). For example, scheduling, qualitative and symbolic reasoning, diagnosis, temporal andspatial planning, hardware design and verification, real-time systems and robot planning. A CSP problem can be considered as of a finite set of variables, each one has a finite domain of values and a set of constraints. A solution to a CSP is a complete assignment of variables which satisfy all constraints. But, finding this solution on a finite domain is a NPcomplete problem requiring a combination of heuristics and combinatory search methods in order to be solved in a reasonable time [1]. In general, approaches to solve CSPs can be classified in two main categories: exact approaches and heuristic ones. As for exact approaches, most of them have the backtracking algorithm (BT) as a main algorithm for solving constraint satisfaction problems. As far as heuristic approaches are concerned, we find a very different approach has taken investigating neural approach works to solve CSP[2]-[4]. In this neural network approach, the constraints are encoded in the network topology, biases strengths connection, and problem is formulated as quadratic cost function which is a Lyapunov function. Particularly, a very different approach has been taken investigating Hopfield network with continuous times for solving CSPs, as we can see in [5]-[8] authors propose mapping CSP to a quadratic model and giving appropriate parameters setting to reach an equilibrium point of $\mathrm{CHN}$. In the practice, there are two important problem with approaches based on conventional neural network architectures, The first is that network is 
partially mitigate the problem of getting stuck in local optimum, the second is due to dynamic Hopfield network which continuously explore the search space and will not stabilize at border 0 or 1 , if the same case appear, we get low solution quality or an incomplete assignment of variables. In order to improve solution or to complete invalid solution, we propose to use MinConflict heuristic [8] after that $\mathrm{CHN}$ reached stabilisation. In this paper we extend our previous study by comparing CHN-MNC with Genetic algorithm [9] and Swarm optimisation [10].

\section{CHN-MNC Algorithm}

CHN-MNC is a collaborative hybrid algorithm, which benefit of the fast convergence of Hopfield neural network and the amelioration of the solution quality by the local search [11]

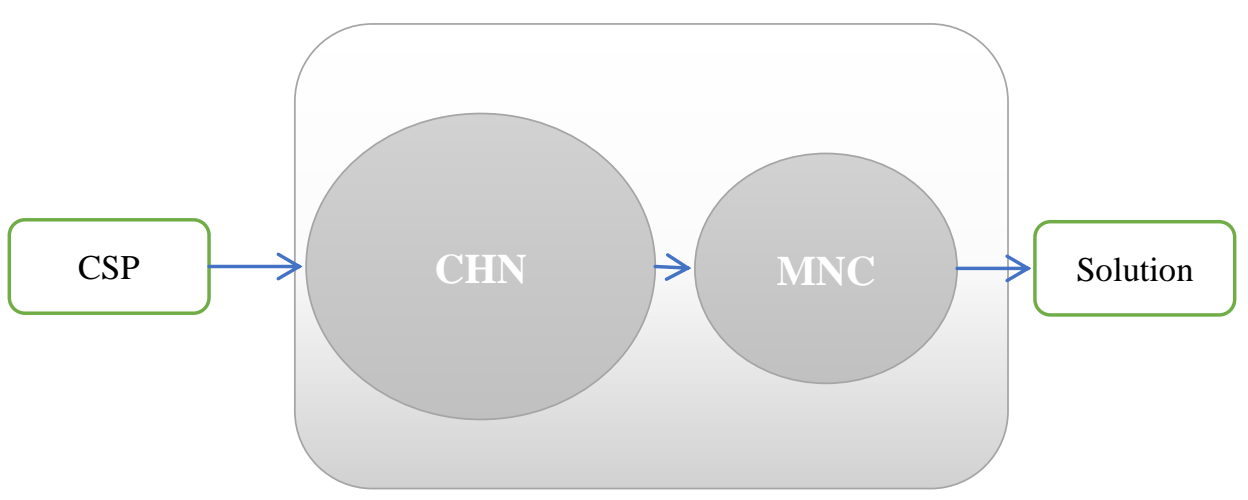

Fig. 1. Architecture CHN-MNC

\subsection{CHN solver}

A large number of real problems such as artificial intelligence, scheduling, assignment problem can be formulated as a Constraint Satisfaction Problem. Solving a CSP requires to finding an assignment of all variables problem under constraints restriction. The CSP can be formulated as three sets [12]:

- Set of $N$ variables $X=\left\{X_{i} ; 1 \leq i \leq N\right\}$.

- Set of $N$ variables domains: $D=\left\{D_{i} ; 1 \leq i \leq d i\right\}$ where each Di contains set of di range values for $\mathrm{X}_{\mathrm{i}}$.

- Set of $M$ constraints: $C=\left\{C_{i} ; 1 \leq i \leq M\right\}$. 
Each constraint $C_{i}$ associates an ordered variables subset which is called the scope of $C_{i}$. The arity of a constraint is the number of involved variables. We can easily reformulate CSP as a Quadratic Problem (QP), by introducing a binary variable $\mathrm{x}_{\mathrm{ik}}$ for each CSP variable $\mathrm{x}_{\mathrm{i}}$, where $\mathrm{k}$ varies over the range of $\mathrm{xi}$, given as follows:

$$
x_{i k}=\left\{\begin{array}{lc}
1, & \text { if variable } \text { i takes value } k \\
0, & \text { otherwise }
\end{array}\right.
$$

For each binary constraint $C_{i j}$, between the variables $y_{i}$ and $y_{j}$, we associate a state function defined as:

$$
S_{i j}(x)=\sum_{r=1}^{d_{i}} \sum_{s=1}^{d_{j}} x_{i r} x_{j s} Q_{i r j s}
$$

Where $\mathrm{x}=\left\{\mathrm{x}_{\mathrm{ik}}, \mathrm{i} \in[1 \ldots \mathrm{N}], \mathrm{k} \in \mathrm{d}_{\mathrm{i}}\right\}$ a vector of QP solution and the quadratic terms Qirjs defined as:

$$
Q_{\text {irjs }}=\left\{\begin{array}{cc}
1 & \text { if }(r, s) \notin C_{i j} \\
0 & \text { otherwise }
\end{array}\right.
$$

From all the equations defined in (2), which correspond to problem constraints, we deduce the objective function of its equivalent $\mathrm{QP}$ :

$$
f(x)=\sum_{i=1}^{N} \sum_{r=1}^{d_{i}} \sum_{j=1}^{N} \sum_{s=1}^{d_{j}} x_{i r} x_{j s} Q_{i r j s}
$$

Furthermore, some strict linear constraints equations must be satisfied by the solution: $\sum_{r=1}^{d_{i}} x_{i r}=1$, for $i=1 . . N$ which can be written also as $A x=b$ ( $A$ is a $N \times M$ matrix and $b$ is a $\mathrm{M}$ dimension vector fully initialized to 1$)$. So, the model is given as follows:

$$
(Q P) \quad\left\{\begin{array}{cc}
\text { Min } & f(x)=x^{T} Q x \\
\text { with } & \\
& A x=b \\
& x \in\{0,1\}^{n}
\end{array}\right.
$$

Systematically, to solve the last Quadratic Optimization Problem with Hopfield model, we need to build an energy function such as the feasible solutions of the problem corresponding to the minimal of $\mathrm{CHN}$ energy function:

$$
E(x)=\frac{\alpha}{2} \sum_{i=1}^{N} \sum_{j=1}^{N} \sum_{r=1}^{d_{i}} \sum_{s=1}^{d_{j}} x_{i r} x_{j s} Q_{i j r s}+\beta \sum_{i=1}^{N} \sum_{r=1}^{d_{i}} x_{i r}+\gamma \sum_{i=1}^{N} \sum_{r=1}^{d_{i}} x_{i r}\left(1-x_{i r}\right)
$$

2.2 MNC local search 
There are many methods which combine two or more no exacts approaches to solve a given optimization problem [13]-[16]. In the same direction we introduce a hybrid approach based CHN and MNC. The MNC algorithm [11] is a very simple and fast local repairing method to resolve CSPs, which aims at assigning all the variables randomly. Next, it iteratively selects one variable from the set of the variables with conflicts which violates one or more constraints of the CSP. Then, it assigns a value to the selected variable, so that it can minimize the number of conflicts. MNC has demonstrated to be able to solve the queens problem in minutes [17]. MNC is widely used to construct hybrid algorithms with other optimizations [14][15][18][19]. In this way, the basic idea of our proposed approach is to use MNC to improve the solution reached by CHN. This will be done in tw o steps (see Figure 2). First, MNC visits all assigned variables; for each one, we apply Min-Conflict directly to the neural network structure, then, it returns the best assignment for the current variable (see Figure 3), the decision will be taken by the sum of all activated neurons weight. Second, we propagate this assignment to other set variables not yet assigned iteratively by applying the MNC heuristic to guarantee as much consistency as possible. The diagram of our proposed algorithm is described by (Figure 4).

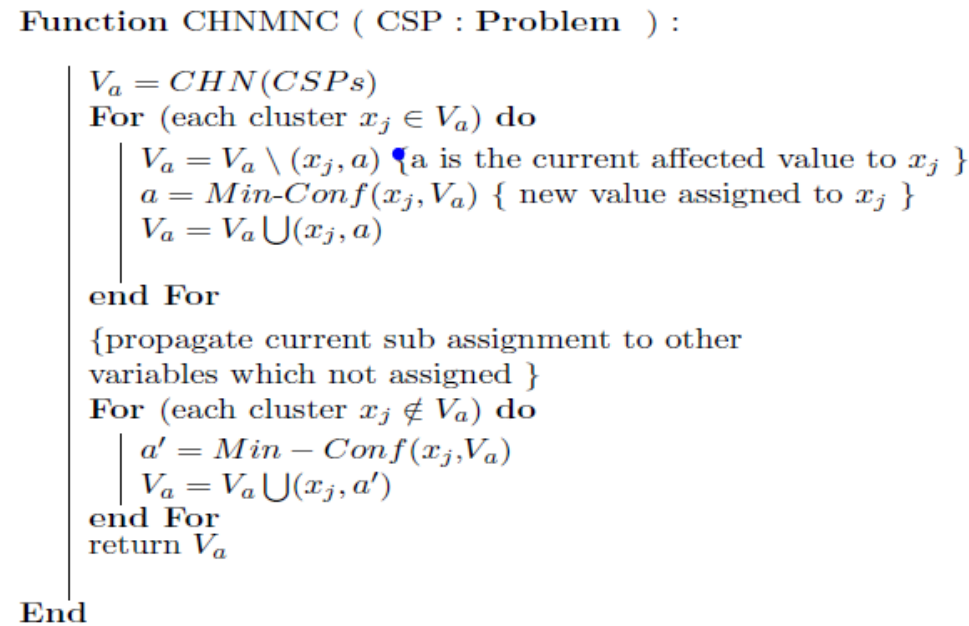

Fig. 2.Main function which improve solution by Min-conflict algorithm 


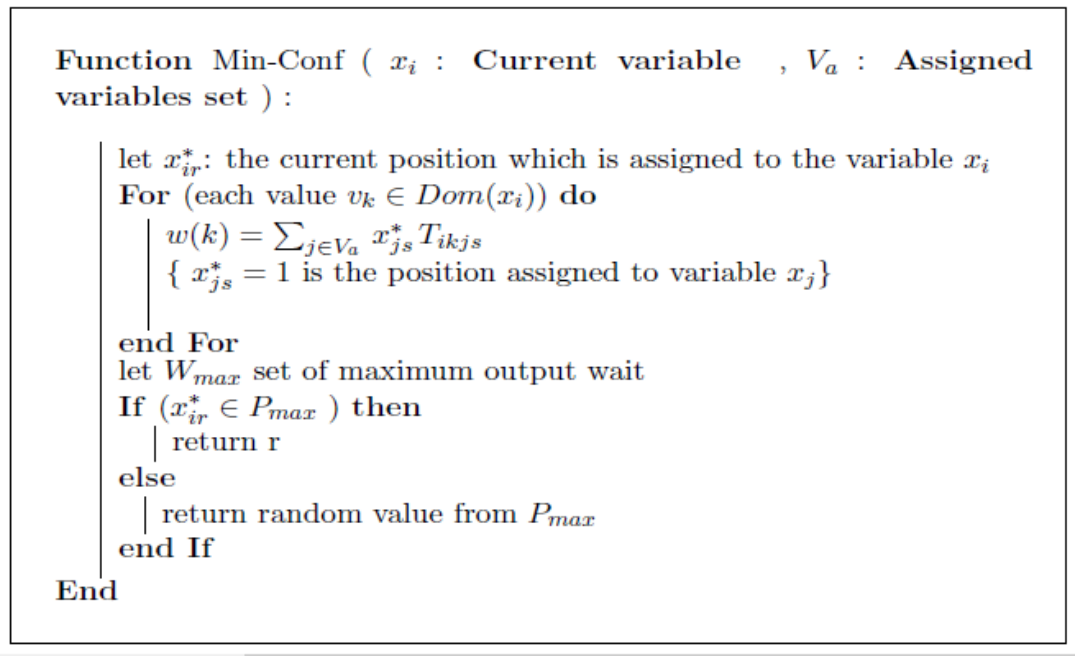

Fig. 3. Selected the most coherent neuron of current cluster with other variables clusters already affected

\section{Evolutionary Algorithm}

The main problem with investing metaheuristic to resolve optimization problem is the probability of becoming trapped at locally optimal points. So, the combination and the cooperation between these approaches may improve the solution quality. Many hybrids approaches have been developed, for our case we shoos two intended, approaches, for solving CSP. The first, one is a combination with genetic algorithm and heuristics reproduction operators. The second is an adapted Swarm optimization to combinatory probes.

\subsection{GA}

We focus on hybrids algorithms which hybrids genetic algorithm with local search. Most all approaches use an integrative hybrid [14]-[16]. So, the Genetic algorithm is executed to the exploration of solution space and local search is used to improve the quality of each intermediate generation of population individual. Others make change on reproduction to adapted GA to a specific problem. In this work we opted for comparison with approach in [20]. The last method use a hybrid search approach that combines the genetic algorithm with the min-conflicts hill-climbing (MCHC).

\subsection{Swarm}

Collective intelligence refers to the capabilities of a resulting community of interactions multiple members (or agents) of the community. Agents can thus accomplish complex tasks through a fundamental mechanism called synergy. Under special conditions, the synergy created by the collaboration between individuals brings out the possibilities of representation, creation and learning superior to those of isolated individuals. The PSO parameters are specific to the problem to be solved and must be determined in each problem. The first work 
that employed the PSO for the resolution of the binary CSP problem was by Schoofs in [21]. The CSP problem is a combinatorial problem, where the concept of speed must be redefined. Thus, we present the redefinition of the usual operators of calculation (sum and product) between the positions and the speeds.

\section{Numerical result}

For showing the practical interest of our approach, we compares it its performance with others metaheuristics approaches over problems of different natures (random, academic and real-world problems). So we choose two algorithms elaborated specially to solve CSP [20], [21]. To perform a competitive comparison we do not settle for the authors original settings, thus we determined them empirically: for GA the population was 200 mutation rate equal to $5 \%$ and crossing rate equal to $72 \%$, as for PSO [21] we choose $\varphi_{1}=\varphi_{2}=1$ and population size fixed at 100 . We run also 500 times each one.

We run some preliminary experiments on the randomly generated problems and we study the performances evolutions of the cited above approaches. We use a random generator based extended model B as it is described in [22]-[24]. This extended model which is called Model $\mathrm{RB}$ is able to generate a hard instance with forced mode which allow that instance have a solution. For each tightness value we generate 100 instances. From Figure 4 we can learn that our approach gives a good solution quality whatever the difficulty (Tightness) of the problem.

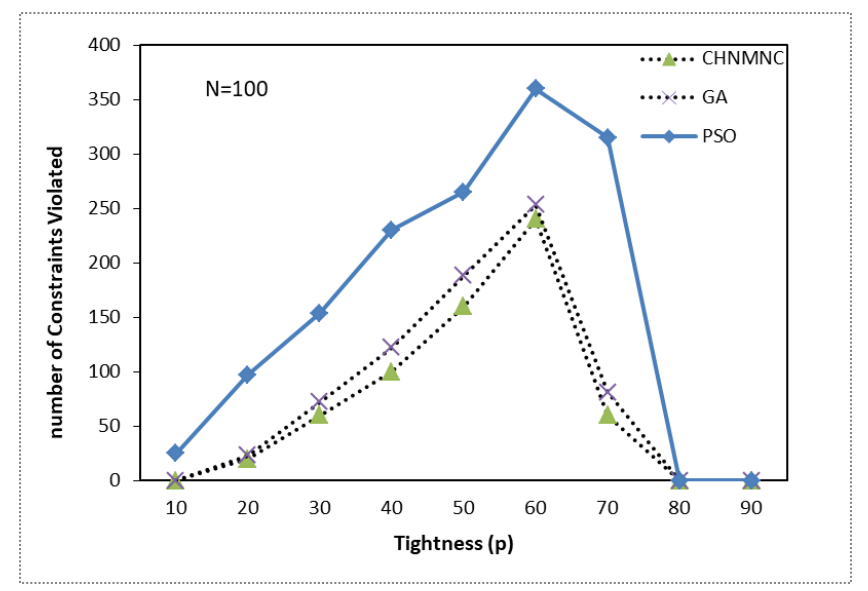

Fig. 4.Performance of GA, PSO and CHN-MNC random gendered instances.

Furthermore, Table 1 shows the results of CHN-MNC, PSO and Genetic Algorithm over 500 independent runs. The simulation is done on selected reals and academics instances of the benchmark [25]. According to the obtained means values our approach better and the standard deviation is more closed to the means values. 
Table 1. Means values and standard deviation performed by CHN-MNC, GA, and PSO over selected instances.

\begin{tabular}{|c|c|c|c|c|c|c|c|c|}
\hline \multirow{2}{*}{$\begin{array}{l}\text { Name of } \\
\text { Instance }\end{array}$} & \multirow[b]{2}{*}{ V } & \multirow[b]{2}{*}{$\mathrm{C}$} & \multicolumn{2}{|c|}{ CHN-MNC } & \multicolumn{2}{|c|}{ GA } & \multicolumn{2}{|c|}{ PSO } \\
\hline & & & Mean & SD & Mean & SD & Mean & $\mathrm{SD}$ \\
\hline queens-10 & 10 & 45 & 1,01 & 0,16 & 2,00 & 1,02 & 13,50 & 5.5 \\
\hline queens-20 & 20 & 190 & 2,40 & 0,32 & 4,00 & 1,06 & 39,20 & 3,11 \\
\hline queens-5-5-5 & 25 & 160 & 0,00 & 0,00 & 0,00 & 0,00 & 19,00 & 2,10 \\
\hline frb30-15-5-mgd & 30 & 210 & 10,00 & 1,07 & 14,00 & 3.46 & 40,10 & 4,08 \\
\hline geom-30a-5 & 30 & 81 & 1,05 & 0,11 & 2,00 & 2,13 & 7,00 & 0,21 \\
\hline geom-30a-6 & 30 & 81 & 0,00 & 0,00 & 0,00 & 0,00 & 4,00 & 0,20 \\
\hline queens-30 & 30 & 435 & 4,00 & 0,94 & 6,00 & 1,05 & 83,00 & 7,39 \\
\hline frb40-19-3-mgd & 40 & 308 & 14,00 & 0,93 & 21,00 & 2,14 & 53,00 & 3,56 \\
\hline geom-40-2 & 40 & 78 & 23,00 & 0,01 & 24,00 & 0,03 & 28,00 & 5,04 \\
\hline geom-40-6 & 40 & 78 & 0,00 & 0,00 & 0,00 & 0,21 & 4,01 & 1,06 \\
\hline myciel-5g-3 & 47 & 236 & 10,00 & 0,22 & 12,00 & 0,50 & 47,00 & 6,83 \\
\hline myciel-5g-4 & 47 & 236 & 5,00 & 0,41 & 8,00 & 0,95 & 29,00 & 3,58 \\
\hline myciel-5g-5 & 47 & 236 & 1,00 & 0,47 & 3,00 & 1,09 & 12,00 & 2,81 \\
\hline myciel-5g-6 & 47 & 236 & 0,00 & 0,13 & 1,00 & 0,31 & 12,50 & 1,51 \\
\hline driverlogw-01c & 71 & 217 & 0,00 & 0,00 & 0,00 & 0,00 & 3,00 & 0,59 \\
\hline composed-25-10-20-5 & 105 & 620 & 13,10 & 1,93 & 14,00 & 6,75 & 56,00 & 7,20 \\
\hline dsjc-125-1-4 & 125 & 736 & 50,00 & 2,30 & 64,00 & 3,69 & 102,00 & 4,15 \\
\hline dsjc-125-1-5 & 125 & 736 & 19,00 & 1,80 & 29,00 & 1,83 & 85,00 & 4,04 \\
\hline Qw h-15-106-1 & 225 & 2324 & 20,00 & 1,60 & 23,00 & 3,68 & 66,60 & 5,11 \\
\hline qwh-15-106-4 & 225 & 2324 & 18,20 & 1,06 & 22,00 & 9,35 & 59,04 & 10,52 \\
\hline qwh-15-106-6 & 225 & 2324 & 22,00 & 2,31 & 23,00 & 5,31 & 68,00 & 8,82 \\
\hline driverlogw-04c & 272 & 3876 & 3,00 & 2,32 & 5,00 & 2,33 & 11,00 & 3,75 \\
\hline driverlogw-02c & 301 & 4055 & 3,00 & 1,17 & 5,00 & 1,89 & 9,00 & 2,01 \\
\hline qwh-20-166-0 & 400 & 5092 & 30,00 & 2,79 & 32,00 & 3,63 & 93,03 & 6,12 \\
\hline qwh-20-166-3 & 400 & 5092 & 29,00 & 1,88 & 31,00 & 2,63 & 89,00 & 4,28 \\
\hline qwh-20-166-6 & 400 & 5092 & 25,00 & 2,19 & 29,00 & 6,84 & 86,00 & 9,27 \\
\hline le-450-5a-3 & 450 & 5714 & 1173,00 & 5,69 & 1261,00 & 767,49 & 1566,00 & 17,03 \\
\hline le-450-5a-4 & 450 & 5714 & 712,00 & 1,89 & 745,00 & 8,95 & 1066,00 & 14,86 \\
\hline le-450-5a-5t & 450 & 5714 & 441,00 & 2,29 & 470,00 & 5,26 & 874,00 & 13,72 \\
\hline
\end{tabular}

\section{Conclusion}

In the last decade several areas have applied CSP model. So, many exact and heuristic methods were introduced. In this logic we focussed our contribution to develops a new hybrid approach based Hopfield neural network and local search. The main contribution in this work is the amelioration by the of the solution given by CHN Some numerical examples assess the effectiveness of the theoretical results are shown in this paper, and also the advantages of this new approach which improve considerably the solution quality and avoid network crush. Other studies are in progress to apply this approach to many problems such as timetabling and resource allocation. 


\section{References}

[1] Carvalho, A. and Santos, C.: A Generator of Heavy-Tailed Search Trees, in Recent Developments in Modeling and Applications in Statistics, Springer. pp. 107-113 (2013)

[2] Tsang, E. P. K. and Wang, C. J.: A generic neural network approach for constraint satisfaction problems. in Neural network applications. Springer.pp. 12-22 (1992).

[3] Adorf , H. M.and Johnston, M. D. :A discrete stochastic neural network algorithm for constraint satisfaction problems. in Neural Networks. IJCNN. pp. 917-924 (1990)

[4] Nakano, T and Nagamatu, M. :Lagrange neural network for solving csp which includes linear inequality constraints, in Artificial Neural Networks: Formal Models and Their Applications-ICANN 2005. Springer. pp. 943-948 (2005)

[5] Bouhouch, A. Loqman, C and El Qadi, A. :Improving CHN by Min-Conflict algorithm to solve constraint satisfaction problems. Adv. Inf. Technol. Theory Appl, vol. 1, no. 1 (2016).

[6] Bouhouch, A. Loqman, C and El Qadi, A. :Scheduling meeting solved by neural network and Min-conflict heuristic, 4th IEEE International Colloquium on in Information Science and Technology (CiSt). pp. 773-778 (2016)

[7] Bouhouch, A. Loqman, C and El Qadi, A. :CHN and Min-Conflict Heuristic to Solve Scheduling Meeting Problems, in Bioinspired Heuristics for Optimization, Springer, pp. 171-184 (2019)

[8] Bouhouch, A. Loqman, C and El Qadi, A. :CHN and Min-Conflict Heuristic to Solve Scheduling Meeting Problems, Meta'2016 Marakech (2016)

[9] Loukakis, E. :A new backtracking algorithm for generating the family of maximal independent sets of a graph. Comput.Math.withAppl, vol. 9, no. 4, pp. 583-589 (1983).

[10] Kanoh, H. Matsumoto, M and Nishihara, S. :Genetic algorithms for constraint satisfaction problems. IEEE International Conference on in Systems, Man and Cybernetics, Intelligent Systems for the 21st Century, vol. 1, pp. 626-631 (1995)

[11] Minton, S. Philips, A. Johnston, M. D. Laird, and P. :Minimizing conflicts: A heuristic repair method for constraint-satisfaction and scheduling problems. J. Artif. Intell, Res., vol. 1, pp. 1-15, (1990)

[12] Montanari,U. :Networks of constraints: Fundamental properties and applications to picture processing. Inf. Sci. (Ny)., vol. 7, pp. 95-132 (1974)

[13] Bouhouch, A. Loqman, C and El Qadi, A. ;CHN and Swap Heuristic to Solve the Maximum Independent Set Problem. International Journal of Electrical and Computer Engineering , vol. 7, no. 6, pp. 3583-3592 ( 2017)

[14] Handa, H. : Hybridization of estimation of distribution algorithms with a repair method for solving constraint satisfaction problems, in Genetic and Evolutionary Computation Conference, pp. 991-1002 (2003)

[15] Liu, T. Liu, M. Zhang, Y.B and Zhang, L. :Hybrid genetic algorithm based on synthetical level of resource conflict for complex construction project scheduling problem. Proceedings of the International Conference on Machine Learning and Cybernetics, vol. 9, pp. 5699-5703. (2005)

[16] Duan, Q. T. Liao, W. and Yi, H. Z. A comparative study of different local search application strategies in hybrid metaheuristics Appl. Soft Computing, vol. 13, no. 3, pp. 1464-1477 (2013)

[17] Minton,S. Johnston, M. D. Philips, A. B and Laird, P ;Minimizing conflicts: a heuristic repair method for constraint satisfaction and scheduling problems. Artif.Intell., vol. 58, no. 1, pp. 161-205 (1992)

[18] Hayakawa, D. Mizuno, K. H. Sasaki, and S. Nishihara, Improving Search Efficiency Adopting Hill-Climbing to Ant Colony Optimization for Constraint Satisfaction Problems, in Knowledge and Systems Engineering (KSE), 2011 Third International Conference on, 2011, pp. 200-204. (2011)

[19] H. Zou and B. Y. Choueiry, : Characterizing the Behavior of a Multi-Agent Search by Using it to Solve a Tight, Real-World Resource Allocation Problem in Workshop on Applications of Constraint Programming, Kinsale, County Cork, Ireland, 2003, pp. 81-101 (2003)

[20] A. E. Eiben, P.-E.Raué, and Z. Ruttkay, : Solving constraint satisfaction problems using genetic algorithms. in Evolutionary Computation, 1994. IEEE World Congress on Computational Intelligence., Proceedings of the First IEEE Conference on, 1994, pp. 542-547 (1994) 
[21] L. Schoofs and B. Naudts : Swarm intelligence on the binary constraint satisfaction problem. in Evolutionary Computation, 2002. CEC'02. Proceedings of the 2002 Congress on, 2002, vol. 2, pp. 1444-1449 (2002)

[22] K. Xu and W. Li, "Exact phase transitions in random constraint satisfaction problems," J. Artif.Intell. Res. (2000)

[23] K. Xu, F. Boussemart, F. Hemery, and C. Lecoutre, A simple model to generate hard satisfiable instances, arXivPrepr.cs/0509032 (2005).

[24] K. Xu, F. Boussemart, F. Hemery, and C. Lecoutre, :Random constraint satisfaction: Easy generation of hard (satisfiable) instances. Artif.Intell., vol. 171, no. 8, pp. 514-534 (2007). 